\title{
Spin-precession-assisted supercurrent in a superconducting quantum point contact coupled to a single-molecule magnet
}

\author{
C. Holmqvist and W. Belzig \\ Fachbereich Physik, Universität Konstanz, D-78457 Konstanz, Germany
}

M. Fogelström

Department of Microtechnology and Nanoscience - MC2, Chalmers University of Technology, SE-412 96 Göteborg, Sweden

(Received 27 February 2012; revised manuscript received 7 June 2012; published 27 August 2012)

\begin{abstract}
The supercurrent through a quantum point contact coupled to a nanomagnet strongly depends on the dynamics of the nanomagnet's spin. We employ a fully microscopic model to calculate the transport properties of a junction coupled to a spin whose dynamics is modeled as Larmor precession brought about by an external magnetic field and find that the dynamics affects the charge and spin currents by inducing transitions between the continuum states outside the superconducting gap region and the Andreev levels. This redistribution of the quasiparticles leads to a nonequilibrium population of the Andreev levels and an enhancement of the supercurrent which is visible as a modified current-phase relation as well as a nonmonotonous critical current as function of temperature. The nonmonotonous behavior is accompanied by a corresponding change in spin-transfer torques acting on the precessing spin and leads to the possibility of using temperature as a means to tune the back-action on the spin.

DOI: 10.1103/PhysRevB.86.054519

PACS number(s): 74.50.+r, 75.76.+j, 75.50.Xx, 75.78.-n
\end{abstract}

\section{INTRODUCTION}

Spintronics devices, which utilize the spin degree of freedom, have already revolutionized the read-out technology used in hard drives. ${ }^{1,2}$ In conventional spintronics, the transport properties of a device typically depend on the relative orientation of the spins of electrons with respect to a reference, which may be a magnetic field or a magnetization direction of a ferromagnetic layer. ${ }^{3}$ The challenge of downsizing electronic devices has lead to the study of transport properties of nonmagnetic single-molecule devices such as diodes ${ }^{4}$ and transistors ${ }^{5-7}$ as well as devices containing single-molecule magnets (SMMs). ${ }^{8}$ The interest in SMMs stems from their long relaxation times at low temperatures ${ }^{9}$ and their display of a wide range of quantum physics phenomena. ${ }^{9,10}$ Studies on SMM devices include for instance three-terminal devices,${ }^{11-17}$ supramolecular spin valves, ${ }^{18}$ and inelastic tunneling spectroscopy. ${ }^{19}$

Interesting spin phenomena may occur when ferromagnets are combined with superconductors (see Ref. 20 and references therein). Cooper pairs in a conventional superconductor have spin-singlet pairing, which, if the superconductor is interfaced with a ferromagnet, extend into the ferromagnet. However, the exchange field inside the ferromagnet tries to align the two spins of the Cooper pairs and hence breaks the Cooper pairs apart resulting in a rapid decay of the superconducting correlations inside the ferromagnet. For the same reasons, the critical current of a Josephson junction with a ferromagnetic layer sandwiched between the two superconductors decays rapidly with increasing thickness of the ferromagnetic layer. ${ }^{21-24}$ On the other hand, if weakly ferromagnetic interfaces with magnetization directions differing from the magnetization direction of the ferromagnetic layer are inserted, the spin-singlet correlations may be transformed into spin-triplet correlations that can survive over a long range within the ferromagnet layer. ${ }^{25-29}$ As a result of this noncollinear magnetization of the ferromagnetic layer, the critical current decays similarly to a supercurrent in a nonmagnetic metal with increasing junction length. ${ }^{30,31}$ Also, the interaction between spin and charge supercurrents has attracted attention. ${ }^{32-35}$ Recently, interest in coupling between the dynamics of magnetic moments and Josephson currents has increased. ${ }^{36-41}$

In Refs. 42 and 43, it was found that spin-singlet to spintriplet conversion can be generated by a nanomagnet such as an SMM or a ferromagnetic nanoparticle coupled to a Josephson junction ${ }^{44}$ provided that the magnetization direction of the nanomagnet precesses. ${ }^{45}$ The spin-triplet correlations enable spin currents to exist close to the junction interface despite the $s$-wave nature of the leads. The spin currents generate a spin-transfer torque acting on the nanomagnet and its effect may be measurable in a ferromagnetic resonance (FMR) experiment $^{46}$ as was suggested in Ref. 42. References 42 and 43 focused on charge and spin currents as well as spin-triplet correlations at zero temperature and in equilibrium junctions. Here, we instead investigate the effects of the spin precession on the critical current and find that the critical current is enhanced at high temperatures. This enhancement is due to a redistribution of the population of states caused by the precessing spin and is also visible in the current-phase relation (CPR). In this paper, we also show the direct relation between the induced equal-spin anomalous Green's functions and the spin current at zero temperature. The spin currents and induced spin-triplet correlations are affected by the redistribution of quasiparticles and change abruptly as a function of temperature when the critical current is enhanced.

The paper is organized as follows: in Sec. II, the model of the superconducting point contact containing the molecular magnet is described as well as the approach to solving the spin-active boundary condition created by the molecular magnet. The results are discussed in Sec. III starting with the Andreev levels and their population. Then, the charge and spin currents are considered along with the induced spin-triplet correlations associated with the spin currents. Finally, the results are summarized in Sec. IV. The Appendix details some of the calculations in Sec. III. 


\section{MODEL AND APPROACH}

We consider a junction consisting of two superconducting leads coupled over a nanomagnet, schematically depicted in Fig. 1(a). ${ }^{42,43}$ The nanomagnet may be an SMM or a ferromagnetic nanoparticle and is subjected to an external effective magnetic field, $\boldsymbol{H}$, which couples to the spin, $\boldsymbol{S}$, of the nanomagnet. Here, we treat the spin classically and if the magnetic field is applied at an angle $\vartheta$ with respect to the spin, it starts precessing with the Larmor frequency $\omega_{L}=\gamma|\boldsymbol{H}|$, where $\gamma$ is the gyromagnetic ratio [see Fig. 1(b)]. The dynamics due to the external field as well as any additional torque, $\tau$, can be summarized in an equation of motion for the spin as

$$
\dot{S}=-\gamma \boldsymbol{H} \times \boldsymbol{S}+\boldsymbol{\tau} .
$$

The additional torque, $\boldsymbol{\tau}$, may include damping as well as spin transfer torques due to transport processes. ${ }^{47}$

The two superconducting leads, denoted by left $(L)$ and right $(R)$, are described by BCS Hamiltonians with order parameters $\Delta(T) e^{ \pm i \varphi / 2}$, where “+" ("-") refers the right (left) lead, $\varphi$ is the superconducting phase difference, and $\Delta(T)$ is the gap at temperature $T$. To investigate the effect of the precessing spin on the Josephson effect, we consider the phenomenological tunnel Hamiltonian $\mathcal{H}_{T}=\sum_{k \sigma ; k^{\prime} \sigma^{\prime}} c_{L, k \sigma}^{\dagger} V_{k \sigma ; k^{\prime} \sigma^{\prime}} c_{R, k^{\prime} \sigma^{\prime}}+$ H.c. where the hopping amplitude depends on the instantaneous direction of the spin, $\boldsymbol{S}$, and can be summarized as $V_{k \sigma ; k^{\prime} \sigma^{\prime}}=\left[V_{0} \delta_{\sigma \sigma^{\prime}}+V_{S}(S(t) \cdot \boldsymbol{\sigma})_{\sigma \sigma^{\prime}}\right] \delta\left(k-k^{\prime}\right){ }^{48,49}$ The mat$\operatorname{rix} \boldsymbol{\sigma}=\left(\sigma_{x}, \sigma_{y}, \sigma_{z}\right)$ consists of the Pauli spin matrices.
The tunnel Hamiltonian poses a spin-active, timedependent boundary condition that can be solved using nonequilibrium Green's functions in the quasiclassical approximation. ${ }^{50-52}$ The junction interface can be treated as a single strong impurity that is included in the quasiclassical theory by means of a $t$-matrix equation, ${ }^{53-55}$ which is then used to calculate the transport properties of the junction. ${ }^{56,57}$ The result is a fully microscopic theory that is able to account for effects involving not only the Andreev levels but also the continuum part of the energy spectrum, ${ }^{58,59}$ see Ref. 42 for details. The transport properties can be expressed in terms of incoming (outgoing) propagators, $\breve{g}_{\alpha}^{i(o)}$, describing quasiparticles in lead $\alpha \in(L, R)$ traveling along trajectories toward (away from) the interface. The " " symbol denotes a matrix in Keldysh space. Using these propagators, one can define a spectral matrix current, $\check{j}_{\alpha}=\breve{g}_{\alpha}^{i}-\breve{g}_{\alpha}^{o}$, from which the charge and spin currents can be obtained as

$$
\begin{aligned}
& j_{\alpha}^{\mathrm{ch}}(t)=\frac{e}{2 \hbar} \int \frac{d \varepsilon}{8 \pi i} \operatorname{Tr}\left[\hat{\tau}_{3} \hat{j}_{\alpha}^{<}(\varepsilon, t)\right], \\
& \dot{j}_{\alpha}^{s}(t)=\frac{1}{4} \int \frac{d \varepsilon}{8 \pi i} \operatorname{Tr}\left[\hat{\tau}_{3} \hat{\boldsymbol{\sigma}} \hat{j}_{\alpha}^{<}(\varepsilon, t)\right],
\end{aligned}
$$

where $\hat{\tau}_{3}=\operatorname{diag}(1,-1), \hat{\sigma}=\operatorname{diag}\left(\sigma,-\sigma_{y} \sigma \sigma_{y}\right)$ and "^» denotes a $4 \times 4$ matrix in Nambu space. The lesser (" $<$ ") propagators are given by the retarded (" $R$ "), Keldysh (" $K$ ") and advanced ones (" $A$ ") as $\hat{g}^{<}=\frac{1}{2}\left(\hat{g}^{K}-\hat{g}^{R}+\hat{g}^{A}\right)$. The itinerant electrons generate a spin transfer torque which gives
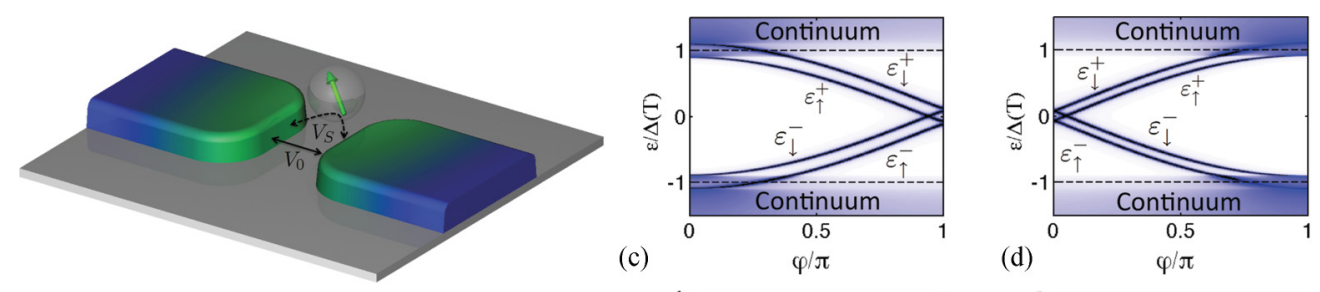

(a)
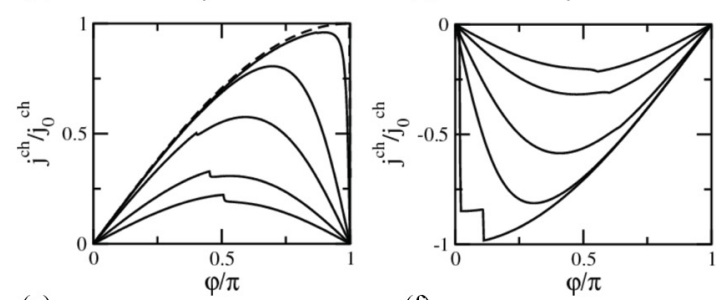

(b)

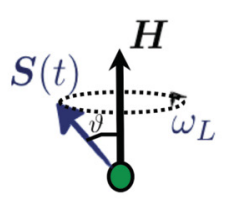

(e)

(f)

FIG. 1. (Color online) (a) Two superconducting leads are coupled over the spin of a nanomagnet. The hopping amplitude for spin-independent tunneling is given by $V_{0}$, while $V_{S}$ is the coupling between the tunneling electrons and the spin of the nanomagnet. (b) The spin of the nanomagnet, $\boldsymbol{S}$, precesses with the Larmor frequency, $\omega_{L}$, due to an external effective magnetic field, $\boldsymbol{H}$, that is applied at an angle $\vartheta$ with respect to the orientation of the spin. The density of states in a rotating frame (see definition in the text) is plotted for a transparent junction $\left(v_{T}=1\right)$ with transmission probabilities (c) $\left(\mathcal{D}_{0}=0.999, \mathcal{D}_{S}=0.025\right)$, given by the hopping amplitudes $\left[v_{0}=v_{T} \cos (0.1 \pi / 2), v_{S}=\right.$ $\left.v_{T} \sin (0.1 \pi / 2)\right]$, and $(\mathrm{d})\left(\mathcal{D}_{0}=0, \mathcal{D}_{S}=1\right)$ given by the hopping amplitudes $\left[v_{0}=v_{T} \cos (\pi / 2)=0, v_{S}=v_{T} \sin (\pi / 2)=1\right]$ at temperature $T / T_{c} \rightarrow 0$. The Andreev levels, which can be seen as sharp states inside the gap, have here for visibility reasons been given an artificial broadening, $0^{+}$, and the continuum density of states is normalized to 1 away from the gap edge. The upper (lower) Andreev levels, $\varepsilon^{+}\left(\varepsilon^{-}\right)$, are given an effective Zeeman splitting $\left(\varepsilon^{+(-)} \rightarrow \varepsilon_{\uparrow, \downarrow}^{+(-)}\right)$by the spin precession. The spin precession couples the Andreev levels and the continuum states as well as the states $\varepsilon_{\uparrow}^{+(-)}$and $\varepsilon_{\downarrow}^{+(-)}$. (e) The current-phase relation $(\mathrm{CPR})$ is plotted for $\left(\mathcal{D}_{0}=0.999, \mathcal{D}_{S}=0.025\right)$ at temperatures $($ solid lines from top to bottom) $T / T_{c}=7.5 \times 10^{-4}, 0.25,0.50,0.75$, and 0.85 . The dashed line shows the CPR for $\left(\mathcal{D}_{0}=1, \mathcal{D}_{S}=0\right)$ at temperature $T / T_{c} \rightarrow 0$. (f) The CPR for a junction with $\left(\mathcal{D}_{0}=0, \mathcal{D}_{S}=1\right.$ ) at temperatures (from bottom to top) $T / T_{c}=7.5 \times 10^{-4}, 0.25,0.50,0.75$, and 0.85 . In (e) and (f), the current is given in units of $j_{0}^{\text {ch }}=e \Delta / \hbar$, where $\Delta$ is the superconducting gap at $T=0$. Figures (c)-(f) are calculated for precession angle $\vartheta=\pi / 4$ and precession frequency $\omega_{L} / \Delta=0.2$. 
a contribution to the torque $\boldsymbol{\tau}$ in Eq. (1) and is given by $\boldsymbol{\tau}=\boldsymbol{j}_{L}^{s}-\boldsymbol{j}_{R}^{s}$.

As in Refs. 42 and 43 , the boundary condition can be simplified by the application of the unitary transformation $\hat{\mathcal{U}}(t)=\operatorname{diag}\left(\mathrm{e}^{-i \frac{\omega_{L}}{2} t \sigma_{z}}, \mathrm{e}^{i \frac{\omega_{L}}{2} t \sigma_{z}}\right)$, which results in a transformation to a rotating frame of reference where the Fermi surfaces of the spin-up and -down bands are shifted by $\mp \omega_{L} / 2$. Note that this transformation also shifts the gap edges; the upper gap edge, $\Delta^{+}(T)=\Delta(T)$, is shifted as $\Delta^{+}(T) \rightarrow \Delta_{\uparrow, \downarrow}^{+}(T)$, where $\Delta_{\sigma}^{+}(T)=\Delta(T)-\sigma \omega_{L} / 2$ and $\sigma=1(-1)$ for spin $\uparrow(\downarrow)$. The lower gap edge, $\Delta^{-}(T)=-\Delta(T)$, is correspondingly modified as $\Delta^{-}(T) \rightarrow \Delta_{\uparrow, \downarrow}^{-}(T)$, where $\Delta_{\sigma}^{-}(T)=-\Delta(T)-$ $\sigma \omega_{L} / 2$. Note also that the hopping elements in quasiclassical theory are replaced by their Fermi surface limits, i.e., the hopping amplitudes $V_{0}$ and $V_{S}$ are replaced by $v_{0}=\pi N_{F} V_{0}$ and $v_{S}=\pi N_{F} S V_{S}$, where $N_{F}$ is the normal density of states at the Fermi energy.

\section{RESULTS}

Scattering processes between two superconductors may lead to constructive interference and the appearance of Andreev levels. ${ }^{60}$ In the presence of a precessing spin, a tunneling quasiparticle may gain (lose) energy $\omega_{L}$ while simultaneously flipping its spin from down (up) to up (down). These additional tunneling processes lead to a modified Andreev level spectrum whose details depend on a number of parameters; the ratio $v_{0} / v_{S}$ determines whether the junction is in a 0 or a $\pi$ state, depending on if $v_{0} / v_{S}>1$ or $v_{0} / v_{S}<1$, respectively. ${ }^{42,43}$ The Larmor frequency, $\omega_{L}$, determines the amount of energy a quasiparticle may gain or lose during tunneling across the junction. The precession angle, $\vartheta$, determines the amount of scattering between the spin-up and spin-down bands. The population of the Andreev states is modified by the temperature, $T$, but also by the scattering processes generated by the precessing spin. This modification of the Andreev level population at finite temperature is the focus of this paper.

\section{A. Andreev levels}

In general, the Andreev levels in the rotating frame can be described by

$\epsilon_{\sigma}^{ \pm}= \pm \Delta(T) \sqrt{1+\Phi\left(v_{0}, v_{S}, \omega_{L}, \vartheta, \varphi\right)+\Xi_{\sigma}\left(v_{0}, v_{S}, \omega_{L}, \vartheta, \varphi\right)}$,

where an analytical expression for $\Phi$ can be found as

$$
\begin{aligned}
\Phi\left(v_{0}, v_{S}, \omega_{L}, \vartheta, \varphi\right)= & -\mathcal{D}_{0}(\vartheta) \sin ^{2}\left(\frac{\varphi}{2}\right) \\
& -\mathcal{D}_{S}(\vartheta) \cos ^{2}\left(\frac{\varphi}{2}\right)+\left[\frac{\omega_{L}}{2 \Delta(T)}\right]^{2},
\end{aligned}
$$

where the transmission probabilities, $\mathcal{D}_{0}$ and $\mathcal{D}_{S}$, depend on the precession angle $\vartheta$. Defining $\chi=1+2\left(v_{0}^{2}+v_{S}^{2}\right)+$ $\left(v_{0}^{2}-v_{S}^{2}\right)^{2}$, the transmission probabilities can be written as

$$
\mathcal{D}_{0}(\vartheta)=2 v_{0}^{2}\left[\frac{1}{\chi}+\frac{\cos (2 \vartheta)}{\chi-4 v_{S}^{2} \sin ^{2} \vartheta}\right]
$$

and

$$
\mathcal{D}_{S}(\vartheta)=2 v_{S}^{2}\left[\frac{1}{\chi}+\frac{\cos (2 \vartheta)}{\chi-4 v_{S}^{2} \sin ^{2} \vartheta}+\frac{2 v_{S}^{2} \sin ^{2}(2 \vartheta)}{\left(\chi-4 v_{S}^{2} \sin ^{2} \vartheta\right)^{2}}\right] .
$$

From the above expressions, it is clear that the junction is in the 0 state if $v_{0}>v_{S}$, and in the $\pi$ state if $v_{0}<v_{S}{ }^{21}$ The function $\Xi_{\sigma}\left(v_{0}, v_{S}, \omega_{L}, \vartheta, \varphi\right)$ provides a Zeeman splitting and an analytical expression for $\Xi_{\sigma}$ can in principle be obtained, but is too involved to allow for simple analytical analysis. The effects of this term are numerically analyzed below. However, in the limit of $v_{S} \rightarrow 0$, but with an arbitrary precession frequency, Eq. (4) simplifies to

$$
\varepsilon_{\sigma}^{ \pm}= \pm \Delta(T)\left[\sqrt{1-\mathcal{D}_{0}(0) \sin ^{2}\left(\frac{\varphi}{2}\right)}-\sigma \frac{\omega_{L}}{2 \Delta(T)}\right] .
$$

From now on we use $\mathcal{D}_{0}=\mathcal{D}_{0}(0)$ and $\mathcal{D}_{S}=\mathcal{D}_{S}(0)$.

The Andreev levels are visible in the density of states as sharp subgap states. The density of states is time independent and given by

$$
\rho_{\alpha}(\varepsilon, \varphi)=-\frac{1}{8 \pi} \Im\left(\operatorname{Tr}\left\{\hat{\tau}_{3}\left[\hat{g}_{\alpha}^{i, R}(\varepsilon, \varphi)+\hat{g}_{\alpha}^{o, R}(\varepsilon, \varphi)\right]\right\}\right) .
$$

In Fig. 1(c), the density of states is plotted in the limit $v_{S} \ll v_{0}$. The Andreev levels seen in the figure are well described by Eq. (8) and their splitting is $\omega_{L}$. The density of states for a junction with $\left(v_{0}=0, v_{S}=1\right)$ is shown in Fig. 1(d). The Zeeman splitting in this case, $v_{S} \gg v_{0}$, can be approximated with $\sim \omega_{L} \cos \vartheta$

\section{B. Enhancement of the critical current}

Each Andreev level carries a certain amount of current that is weighted by the Andreev level occupation. In equilibrium for a static spin, the amount of current each Andreev level, $\varepsilon^{ \pm}$, carries is $(2 e / \hbar) \partial \varepsilon^{ \pm} / \partial \varphi$ while the population of the quasiparticle states is given by the Fermi distribution function, $\phi_{0} \cdot{ }^{61,62}$ In this equilibrium situation, the lesser propagators entering Eq. (2) can be written as $g_{\alpha, \sigma \sigma}^{i / o,<}(\varepsilon, \varphi)=-2 \pi \rho_{\alpha, \sigma \sigma}^{i / o}(\varepsilon, \varphi) \phi_{0}^{<}(\varepsilon)$ using the partial density of states $\rho_{\alpha, \sigma \sigma^{\prime}}^{i / o}(\varepsilon, \varphi)=\left(-\frac{1}{8 \pi}\right) \mathfrak{\Im}\left[\left(\hat{g}_{\alpha}^{i / o, R}\right)_{\sigma \sigma^{\prime}}(\varepsilon, \varphi)\right]$. Hence, in equilibrium, the charge current for one channel is given by

$$
j^{\mathrm{ch}}(\varphi)=\frac{e \Delta(T)}{2 \hbar} \frac{\left(\mathcal{D}_{0}-\mathcal{D}_{S}\right) \sin (\varphi) \tanh \left[\varepsilon^{+}(\varphi) / 2 T\right]}{\sqrt{1-\mathcal{D}_{0} \sin ^{2}(\varphi / 2)-\mathcal{D}_{S} \cos ^{2}(\varphi / 2)}} .
$$

In a nonequilibrium situation where a superconducting point contact contains a spin precessing with a finite frequency, the current-phase relation is modified. As was shown in Refs. 42 and 43, the Josephson charge current is time independent and in the case of dominating spin-dependent tunneling and zero temperature, the CPR exhibits abrupt jumps as a function the superconducting phase difference, $\varphi$. As the temperature is increased, the abrupt jumps are smoothed out and a new step at a phase difference $\varphi_{p}$ develops as can be seen in panels (e) and (f) of Fig. 1. This step, which consequently gives a peak in the CPR at $\varphi_{p}$, is the result of an enhanced Josephson current at high temperatures for phase differences in the interval $0 \leqslant \varphi \leqslant \varphi_{p}$ for 0 junctions and for $\varphi_{p} \leqslant \varphi \leqslant \pi$ 


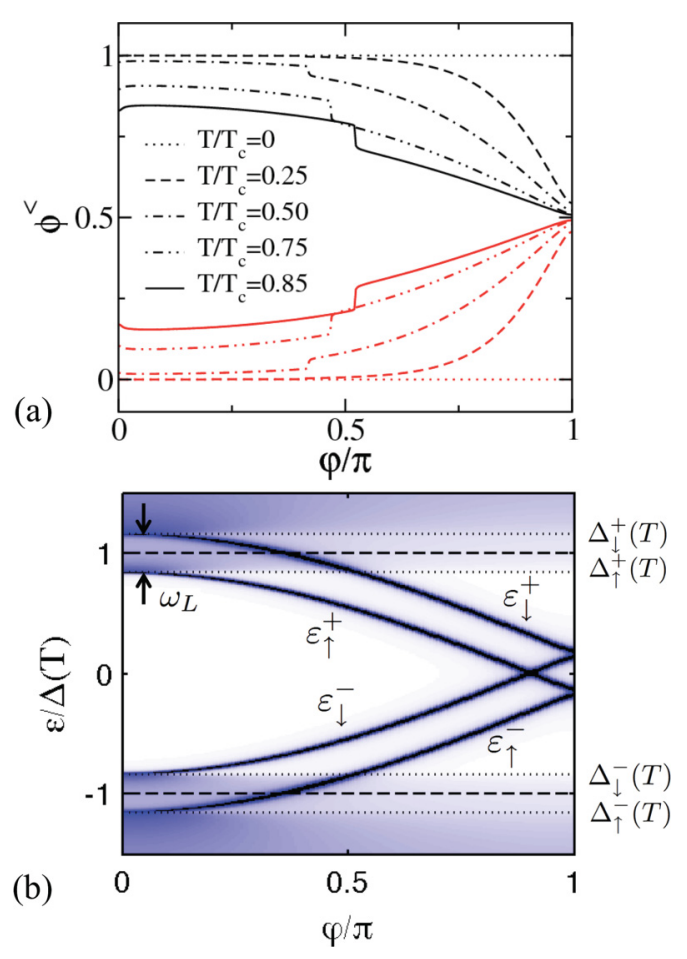

FIG. 2. (Color online) (a) Population of the lower Andreev level $\varepsilon_{\uparrow}^{-}$(black lines) and the upper Andreev level $\varepsilon_{\downarrow}^{+}$(red lines) as function of phase difference, $\varphi$, for a 0 junction with transmission probabilities $\mathcal{D}_{0}=0.999$ and $\mathcal{D}_{S}=0.025$, precession frequency $\omega_{L} / \Delta=0.2$ and tilt angle $\vartheta=\pi / 4$. At temperature $T / T_{c}=7.5 \times 10^{-4}$, the population of the Andreev levels is unaffected by the phase difference. At higher temperatures, $T / T_{c}=0.25,0.50,0.75$, and 0.85 , the population changes abruptly at a phase difference $\varphi_{p}$ leading to a jump in the CPRs shown in Fig. 1. (b) Density of states for the junction in (a) at temperature $T / T_{c}=0.85$. The dashed lines mark $\Delta^{ \pm}(T)=$ $\pm \Delta(T)$. The dotted lines denote $\Delta_{\sigma}^{ \pm}(T)=\Delta^{ \pm}(T)-\sigma \omega_{L} / 2$.

for $\pi$ junctions. To understand this current enhancement, we now turn to the population of the Andreev levels.

The population of the spin band $\sigma$ in a nonequilibrium situation can analogously to the equilibrium case be defined as

$$
\phi_{\sigma}^{i / o,<}(\varepsilon, \varphi)=g_{0, \sigma \sigma}^{i / o,<}(\varepsilon, \varphi) /\left[-2 \pi \rho_{\sigma \sigma}^{i / o}(\varepsilon, \varphi)\right] .
$$

In Fig. 2(a), the occupation of the Andreev levels $\varepsilon_{\uparrow}^{-}$and $\varepsilon_{\downarrow}^{+}$are shown as a function of phase difference. At zero temperature, the lower Andreev level, $\varepsilon_{\uparrow}^{-}$, is fully occupied while the upper Andreev level, $\varepsilon_{\downarrow}^{+}$, is unoccupied. At higher temperatures, the lower Andreev level's occupation is decreased while the upper correspondingly has a finite occupation. In addition, there is an abrupt change in the population corresponding to the jump in the CPR at phase difference $\varphi_{p}$. The abrupt change in the population is an effect of the spin-flip scattering processes in which a quasiparticle interacting with the precessing spin may gain or lose energy $\omega_{L}$. These scattering processes couple the Andreev levels with the continuum states provided that

$$
\omega_{L} \geqslant\left|\Delta_{\sigma}^{+(-)}(T)\right|-\left|\varepsilon_{\sigma}^{+(-)}\right|,
$$

see Fig. 2(b). The coupling causes quasiparticles to be promoted to the lower Andreev level from the continuum below $\Delta_{\sigma}^{-}(T)$. Quasiparticles in the upper Andreev level are similarly scattered into the upper continuum. If the lower

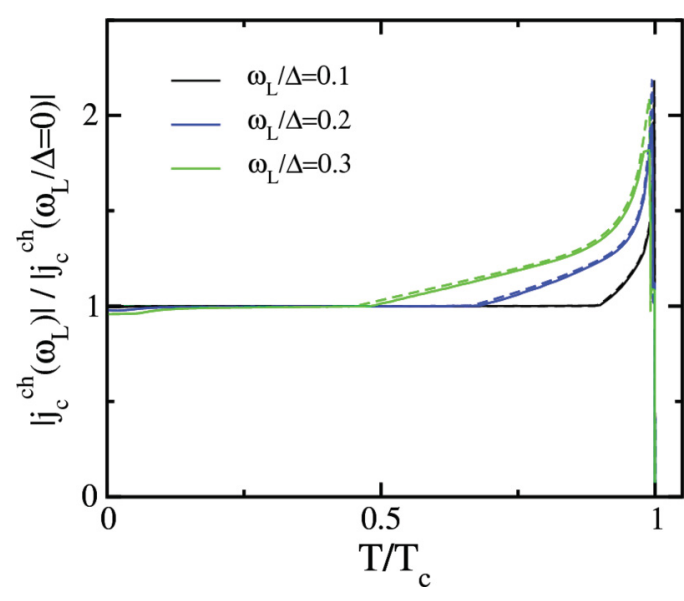

FIG. 3. (Color online) Enhanced critical current as a function of temperature, $T / T_{c}$, for $\left(\mathcal{D}_{0}=0.999, \mathcal{D}_{S}=0.025\right)$ (dashed lines) and $\left(\mathcal{D}_{0}=0, \mathcal{D}_{S}=1\right)$ (solid lines). The enhancement of the spinprecession-assisted critical current $j_{c}^{\text {ch }}\left(\omega_{L}\right)$ is calculated with respect to the critical current through a junction with a static spin and the respective hopping amplitudes specified above, viz. $j_{c}^{\text {ch }}\left(\omega_{L} / \Delta=0\right)$, which is the critical current obtained from Eq. (10). The precession angle is $\vartheta=\pi / 8$.

(upper) Andreev level is not completely filled (unoccupied), which is the case at finite temperature, the coupling leads to a repopulation (emptying) of the Andreev level similarly to the repopulation of Andreev levels due to microwave radiation. ${ }^{58,59}$ This process enhances the supercurrent as is shown in Figs. 1(e) and 1(f). The peak in the CPR at phase difference $\varphi_{p}$ can hence be found from the equality in Eq. (12). In the case of $v_{S} \ll v_{0}$, Eq. (8) can be used to obtain

$$
\varphi_{p}=2 \arcsin \left\{\sqrt{\frac{\omega_{L}}{\mathcal{D}_{0} \Delta(T)}\left[2-\frac{\omega_{L}}{\Delta(T)}\right]},\right.
$$

where $\omega_{L} \leqslant \mathcal{D}_{0} \Delta(T) / 2$. As a consequence, $\varphi_{p}$ increases as the temperature increases and the superconducting gap, $\Delta(T)$, closes.

The enhancement of the charge current for certain phase differences may lead to an enhanced critical current if the enhancement is large enough. Figure 3 shows the temperaturedependent enhancement of the critical charge current. The
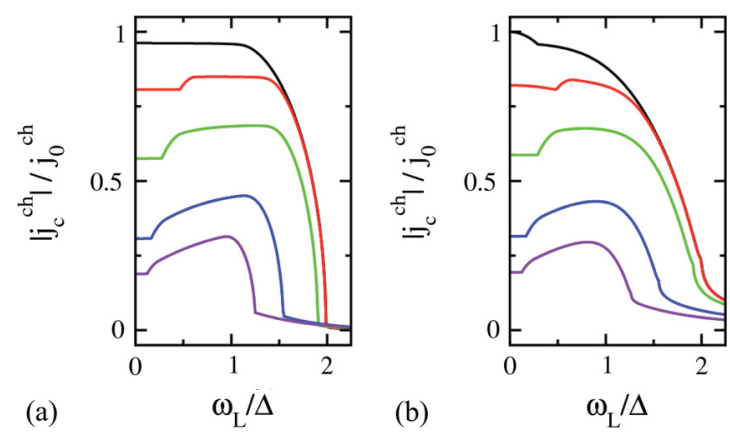

FIG. 4. (Color online) Critical current as function of precession frequency, $\omega_{L}$, for (a) $\left(\mathcal{D}_{0}=0.999, \mathcal{D}_{S}=0.025\right)$ and (b) $\left(\mathcal{D}_{0}=0\right.$, $\mathcal{D}_{S}=1$ ), where the precession angle is taken to be $\vartheta=\pi / 8$ and the temperature is $T / T_{c}=7.5 \times 10^{-4}$ (black), 0.25 (red), 0.50 (green), 0.75 (blue), and 0.85 (violet). 

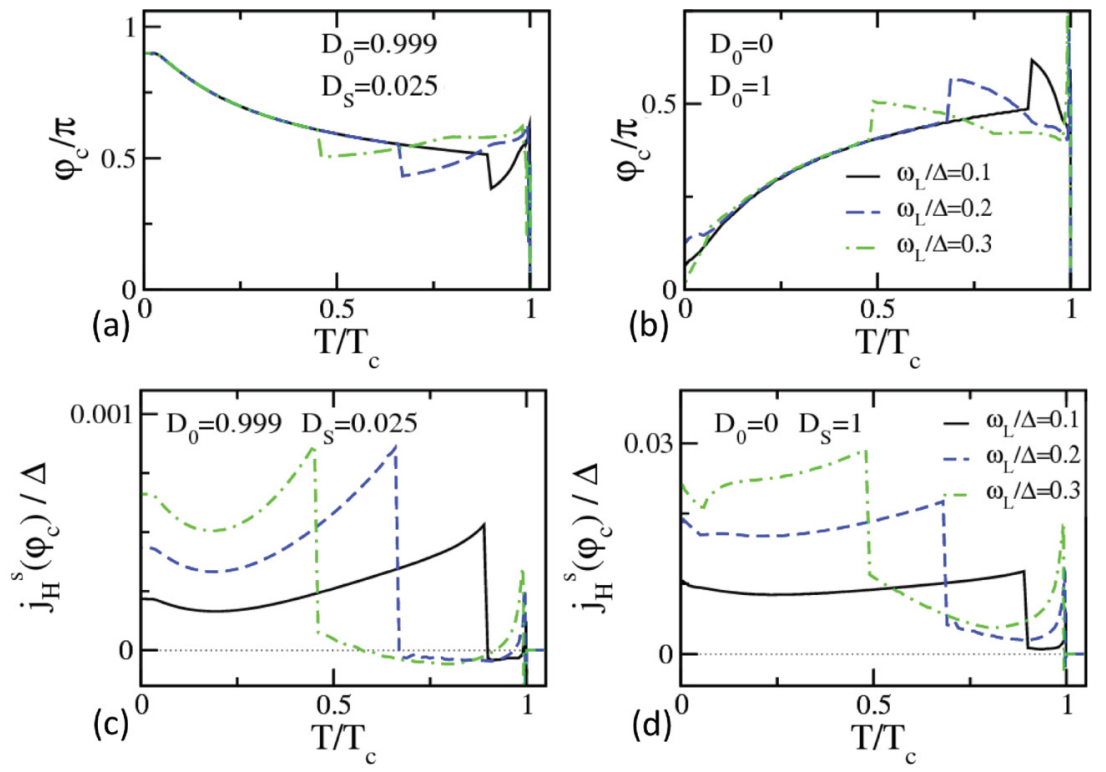

FIG. 5. (Color online) (a) and (b) The superconducting phase difference, $\varphi_{c}$, corresponding to the critical charge current, i.e., $j_{c}^{\mathrm{ch}}=j^{\mathrm{ch}}\left(\varphi_{c}\right)$, is plotted as a function of temperature, $T / T_{c}$, for (a) $\left(\mathcal{D}_{0}=0.999, \mathcal{D}_{S}=0.025\right)$ and (b) $\left(\mathcal{D}_{0}=0\right.$, $\left.\mathcal{D}_{S}=1\right)$ for precession frequencies $\omega_{L} / \Delta=$ $0.1,0.2$, and 0.3 . The abrupt jump in the phase difference occurs when the critical current is given by the spin-precession-enhanced current. The abrupt change in $\varphi_{c}$ leads to an abrupt change in the spin-current component $j_{H}^{s}\left(\varphi_{c}\right)$, which is plotted for hopping strengths $(\mathrm{c})\left(\mathcal{D}_{0}=0.999, \mathcal{D}_{S}=0.025\right)$ and (d) $\left(\mathcal{D}_{0}=0, \mathcal{D}_{S}=1\right)$. The precession angle is $\vartheta=\pi / 8$ in all panels. enhancement of the critical current may be understood as an effective lowering of the temperature due to the repopulation of the lower Andreev levels and the emptying of the upper Andreev levels; the Andreev level population generated by the precessing spin at a certain temperature, $\phi_{\sigma}^{i / o,<}$ $\left(\omega_{L}>0, T_{1}\right)$, corresponds to an Andreev level population at a lower temperature but with zero precession frequency, $\phi_{\sigma}^{i / o,<}\left(\omega_{L}=0, T_{2}<T_{1}\right)$.

The critical charge current as a function of precession frequency is shown in Fig. 4. The critical current is enhanced at high enough precession frequencies due to the redistribution of the quasiparticle occupation. At even higher precession frequencies, quasiparticles can be scattered between the lower (upper) Andreev levels, $\varepsilon_{\sigma}^{-}\left(\varepsilon_{\sigma}^{+}\right)$, and the upper (lower) continuum states, similarly to what was found in Refs. 58 and 59. These processes lead to a decrease of the critical current at precession frequencies $\sim 2 \Delta(T)$.

\section{Spin currents and spin-triplet correlations}

In Refs. 42 and 43, it was found that the spin-dependent Andreev scattering across the junction produces a spin structure of the lead propagators, even at zero temperature. These spin-triplet correlations were in Ref. 42 quantified using $\boldsymbol{d}$ vectors as these are commonly used to characterize the order parameters of spin-triplet superconductors. In this paper, the direct relation between spin currents and spin-triplet correlations is investigated. This can easily be done in the case of a small tilt angle. If the incoming (outgoing) propagator in the case of zero tilt angle is $\check{g}_{\alpha}^{0, i(o)}$, it can be shown that a small tilt angle gives a contribution, $\delta \check{g}_{\alpha}^{i(o)}$, to first order such that $\breve{g}_{\alpha}^{i(o)}=\breve{g}_{\alpha}^{i(o)}+\delta \check{g}_{\alpha}^{i(o)}$ (see the Appendix for details). The first-order contribution to the propagators has the spin structure $\delta \check{g}_{\alpha}^{i(o)}=\delta \breve{g}_{\alpha}^{i(o), \uparrow}+\delta \check{g}_{\alpha}^{i(o), \downarrow}$ where $\uparrow$ and $\downarrow$ denote equal-spin correlation terms. The charge current is identical to its value in the $\vartheta=0$ case and is hence unaffected to first order. Using the symmetry relations for propagators in Keldysh-Nambu space, ${ }^{63}$ the spin current, in terms of only the normal Green's functions, now reads

$$
j_{\alpha}^{s, \uparrow(\downarrow)}(t)=\frac{1}{4} \int \frac{d \varepsilon}{4 \pi i}\left\{\left[\delta g_{\alpha}^{\uparrow(\downarrow), i,<}(\varepsilon, t)-\delta g_{\alpha}^{\uparrow(\downarrow), o,<}(\varepsilon, t)\right]\right\} .
$$

However, the normalization condition, $\breve{g}^{2}=-\pi^{2} \breve{1}$, can be used to relate the anomalous and the normal Green's functions. The relations between the anomalous Green's functions, $f_{\alpha}^{\uparrow(\downarrow)}$, and the normal Green's functions, $g_{\alpha}^{\uparrow(\downarrow)}$, entering into the spin current of Eq. (14), are given in Eqs. (A4) and (A5) in the Appendix. From these relations, it is explicitly shown that the equal-spin anomalous Green's functions $\breve{f}_{\alpha}^{\uparrow / \downarrow}$ induced by the precessing spin determine the spin current. Conversely, the spin current vanishes for $\breve{f}_{\alpha}^{\uparrow / \downarrow} \equiv 0$ at zero temperature. In other words, the spin current is a direct measure of the induced equal spin-triplet correlations.

The induced spin-triplet correlations exist close to the junction interface and decay on the scale of the superconducting coherence length. ${ }^{42,43}$ Hence, the spin current lacks a dc component but instead has a precessing polarization. In particular, it has a term due to the spin-dependent Andreev scattering that can be expressed as $j_{\alpha, H}^{s}(\gamma \boldsymbol{H}) \times S$, which is finite only for temperatures $T<T_{c}$. This term generates a feedback effect on the precessing spin in the form of a spin-transfer torque $^{64,65}$ given by $\boldsymbol{\tau}_{A}=\left(j_{L, H}^{s}-j_{R, H}^{s}\right)(\gamma \boldsymbol{H}) \times \boldsymbol{S} .^{47,66}$ This spin-transfer torque acts as an effective magnetic field and shifts the precession frequency as $\omega_{L} \rightarrow \omega_{L}\left[1+2 j_{H}^{s}\right]$, where $j_{H}^{s}=j_{L, H}^{s}=-j_{R, H}^{s}$. Plotting the magnitude of this spincurrent component in relation to the critical current shows that the spin current, and therefore also the induced spin-triplet correlations, changes dramatically as the critical current is enhanced by the spin precession (see Fig. 5).

\section{CONCLUSIONS}

In summary, we have studied the nonequilibrium transport properties of a superconducting point contact coupled to the precessing spin of a nanomagnet using a fully microscopic model. First, we analyzed the Andreev levels and their 
population as a function of temperature. It was found that the precession of the nanomagnet modifies the Andreev scattering in such a way that it leads to scattering of quasiparticles from the continuum part of the energy spectrum into the lower Andreev levels. These transitions are similar to those occurring in microwave-irrated superconducting quantum point contacts ${ }^{58,59}$ and lead to a nonequilibrium population of the Andreev levels and an effective cooling of the point contact.

We have also shown that the effective cooling leads to an enhanced supercurrent. If the precession frequency of the nanomagnet's spin is large enough, this spin-precession-assisted supercurrent has an enhanced critical current. The enhancement of the critical current increases with increasing temperature.

The scattering across the junction leads to additional nonequilibrium processes: Besides the enhanced supercurrent, we have also shown that spin-triplet correlations are created as a response to the scattering processes generated by the spin precessing at a finite angle. Moreover, we have shown that the induced spin-triplet correlations produce a spin current and that the spin current at zero temperature essentially is a measurement of the induced spin-triplet correlations. As was shown previously, ${ }^{42,43}$ one of the spin current components shifts the nanomagnet's precession frequency. Here, this spincurrent component was studied for the same superconducting phase difference that is associated with the critical current and the magnitude of this component was found to be drastically modified as a function of temperature. The critical current enhancement is an effect of the nonequilibrium processes taking place inside the junction and a measurement of this enhancement would suggest the existence of induced spin-triplet correlations since both phenomena are of the same origin.

A natural question concerns the experimental control over the junction parameters. Aluminum microbridges can be used to fabricate few-channel superconducting atomic point contacts ${ }^{67}$ whose transmission eigenvalues are possible to determine via transport measurements. ${ }^{68,69}$ The superconducting gap in aluminum is $\sim 200 \mu \mathrm{eV}$ but can be made smaller in an atomic point contact. Typical values for precession frequencies are in the range of tenths of $\mathrm{GHz}$ for ferromagnetic-resonance experiments performed on thin ferromagnetic films in contact with superconductors. ${ }^{46}$ With an applied external magnetic field of $\sim 60 \mathrm{mT}$, the corresponding gyromagnetic ratio is close to that of free electrons, $\gamma=2 \mu_{B} / \hbar$. Assuming similar values for the nanomagnet, the precession frequency is in the range $\omega_{L} / \Delta \sim 0.07-0.3$ for a junction with a superconducting gap in the range $\Delta \sim 20-100 \mu \mathrm{eV}$.

A direct measurement of the current-phase relation can in principle be done for atomic point contacts. ${ }^{70}$ However, this type of measurement requires the addition of a superconducting quantum interference device (SQUID) loop to control the phase difference of the superconducting junction and the magnetic flux through the loop might interfere with the magnetic control of the dynamics of the nanomagnet. Therefore measurements of the critical current as a function of temperature should be a more practical route to finding experimental signatures of the coupling between a superconducting junction and the dynamics of a nanomagnet and could lead to more insight into the interplay between superconductivity and ferromagnetism.

\section{ACKNOWLEDGMENTS}

C.H. and W.B. were supported by Deutsche Forschungsgemeinschaft and SFB 767. M.F. acknowledges support from the Swedish Research Council (VR).

\section{APPENDIX: RELATION BETWEEN NORMAL AND ANOMALOUS GREEN'S FUNCTIONS}

In the limit of a small precession angle, the contributions to the $t$ matrices and propagators can be calculated as follows: starting with a junction with zero tilt angle, $\vartheta=0$, the hopping element takes the diagonal form

$$
\hat{v}=\left(\begin{array}{cc}
v_{0}+v_{S} \sigma_{z} & 0 \\
0 & v_{0}+v_{S} \sigma_{z}
\end{array}\right) \equiv \hat{v}^{d}
$$

and the corresponding $t$-matrix equation is given by $\check{t}_{\alpha}^{0}=\check{\Gamma}_{\alpha}^{0}+$ $\check{\Gamma}_{\alpha}^{0} \check{g}_{\alpha}^{0} \breve{t}_{\alpha}^{0}$, where $\check{g}_{\alpha}^{0}$ is the unperturbed Green's function in lead $\alpha$ and $\check{\Gamma}_{L / R}^{0}=\check{v}^{d} \breve{g}_{R / L}^{0} \breve{v}^{d}$. The solution $\check{t}_{\alpha}^{0}$ has a diagonal form and may include nonequal spin-triplet components due to a nonzero $v_{S}$ value. The resulting propagators [see Eq. (3)] yield a spin current $\boldsymbol{j}_{\alpha}^{s}=0$.

If a small tilt angle, $\vartheta \neq 0$, is introduced, the hopping element is modified into $\hat{v}=\hat{v}^{d}+\hat{v}^{\uparrow}+\hat{v}^{\downarrow}$, where $\hat{v}^{d}$ is given by Eq. (A1) and $\hat{v}^{\uparrow(\downarrow)}$ is proportional to $\vartheta$ [disregarding terms of $\left.O\left(\vartheta^{2}\right)\right]$ :

$$
\hat{v}^{\uparrow(\downarrow)} \equiv\left(\begin{array}{cc}
v_{S} \vartheta \sigma_{+(-)} & 0 \\
0 & v_{S} \vartheta \sigma_{-(+)}
\end{array}\right) .
$$

The hopping elements enter into the $t$ matrix equation via $\check{\Gamma}_{L / R}^{0}=\check{v}^{d} \check{g}_{R / L}^{0} \check{v}^{d}$ and $\delta \check{\Gamma}_{L / R}^{\uparrow(\downarrow)}=\hat{v}^{\uparrow(\downarrow)} \check{g}_{R / L}^{0} \hat{v}^{d}+\hat{v}^{d} \check{g}_{R / L}^{0} \hat{v}^{\uparrow(\downarrow)}$. The resulting linearized $t$ matrix can be written as $\check{t}_{\alpha}=$ $\check{t}_{\alpha}^{0}+\delta \check{t}_{\alpha}$, where $\check{t}_{\alpha}^{0}$ is the zero-angle solution and the components of $\delta \check{t}_{\alpha}=\delta \check{t}_{\alpha}^{\uparrow}+\delta \check{t}_{\alpha}^{\downarrow}$ are given by $\delta \check{t}_{\alpha}^{\uparrow(\downarrow)}=$ $\left[\check{1}-\check{\Gamma}_{\alpha}^{0} \check{g}_{\alpha}^{0}\right]^{-1} \delta \check{\Gamma}^{\uparrow(\downarrow)}\left[\check{1}+\check{g}_{\alpha}^{0} \check{t}_{\alpha}^{0}\right]$.

The direction dependence of the propagators can be captured by separating them into incoming or outgoing propagators depending on whether they describe quasiparticles moving towards or away from the junction interface. The incoming and outgoing propagators can be obtained as $\check{g}_{\alpha}^{i / o}=\check{g}_{\alpha}^{0, i / o}+$ $\delta \check{g}_{\alpha}^{i / o}$, where $\delta \check{g}_{\alpha}^{i / o}=\delta \check{g}_{\alpha}^{\uparrow, i / o}+\delta \check{g}_{\alpha}^{\downarrow, i / o}$ and $\delta \check{g}_{\alpha}^{\uparrow(\downarrow), i / o}=\left(\check{g}_{\alpha}^{0} \pm\right.$ $i \pi \breve{1}) \delta \check{t}_{\alpha}^{\uparrow()}\left(\check{g}_{\alpha}^{0} \mp i \pi \breve{1}\right)$. Using the parametrization for the spinsinglet (" $s$ ") and spin-triplet (" $t$ ") components of the normal and anomalous Green's functions:

$$
\begin{aligned}
& g_{s}^{i / o, X}+\boldsymbol{g}_{t}^{i / o, X} \cdot \boldsymbol{\sigma}=\left(\begin{array}{ll}
g_{+}^{0, i / o, X} & \delta g_{\uparrow}^{i / o, X} \\
\delta g_{\downarrow}^{i / o, X} & g_{-}^{0, i / o, X}
\end{array}\right), \\
&\left(f_{s}^{i / o, X}+\boldsymbol{f}_{t}^{i / o, X} \cdot \boldsymbol{\sigma}\right) i \sigma_{y}=\left(\begin{array}{cc}
-\delta f_{\uparrow}^{i / o, X} & f_{+}^{0, i / o, X} \\
-f_{-}^{0, i / o, X} & \delta f_{\downarrow}^{i / o, X}
\end{array}\right),
\end{aligned}
$$

where $X \in(R, K, A)$, and the normalization condition for Keldysh-Nambu Green's functions, the retarded and advanced normal Green's functions can be expressed in terms of the anomalous ones. In the limit of a small tilt angle, the linearized relations between the normal and anomalous Green's functions read

$$
\begin{aligned}
& \delta g_{\uparrow}^{R / A}=\tilde{F}_{-}^{R / A} \delta f_{\uparrow}^{R / A}+F_{+}^{R / A} \delta \tilde{f}_{\uparrow}^{R / A}, \\
& \delta g_{\downarrow}^{R / A}=\tilde{F}_{+}^{R / A} \delta f_{\downarrow}^{R / A}+F_{-}^{R / A} \delta \tilde{f}_{\downarrow}^{R / A},
\end{aligned}
$$


where $F_{ \pm}^{R / A}=f_{ \pm}^{0, R / A} / \xi^{0, R / A}$ and $\tilde{F}_{ \pm}^{R / A}=\tilde{f}_{ \pm}^{0, R / A} / \xi^{0, R / A}$. Here, $g_{ \pm}^{X}$ and $f_{ \pm}^{X}$ are the diagonal matrix components given by $g_{ \pm}^{X}=g_{s}^{X} \pm g_{z}^{X}$ and $f_{ \pm}^{X}=f_{s}^{X} \pm f_{z}^{X}$ and $\xi^{R / A}=g_{+}^{0, R / A}+$ $g_{-}^{0, R / A}$. The off-diagonal Keldysh components are

$$
\begin{aligned}
\delta g_{\uparrow}^{K}= & \delta f_{\uparrow}^{R} \tilde{F}_{-}^{\prime K}+\delta \tilde{f}_{\uparrow}^{A} F_{+}^{\prime K}-\delta \tilde{f}_{\uparrow}^{R} F_{+}^{R} G_{-}^{K}-\delta f_{\uparrow}^{A} \tilde{F}_{-}^{A} G_{+}^{K} \\
& +\delta f_{\uparrow}^{K} \tilde{F}_{-}^{\prime A}+\delta \tilde{f}_{\uparrow}^{K} F_{+}^{\prime R}, \\
\delta g_{\downarrow}^{K}= & \delta f_{\downarrow}^{R} \tilde{P}_{+}^{\prime K}+\delta \tilde{f}_{\downarrow}^{A} P_{-}^{\prime K}-\delta \tilde{f}_{\downarrow}^{R} F_{-}^{R} Q_{+}^{K}-\delta f_{\downarrow}^{A} \tilde{F}_{+}^{A} Q_{-}^{K} \\
& \times \delta f_{\downarrow}^{K} \tilde{P}_{+}^{\prime A}+\delta \tilde{f}_{\downarrow}^{K} P_{-}^{\prime R},
\end{aligned}
$$

where the retarded-type and advanced-type matrices are

$$
\begin{aligned}
& {F_{ \pm}^{\prime R / A}}^{\prime R / A} f_{ \pm}^{0, R / A} / \zeta_{1}, \quad P_{ \pm}^{\prime R / A}=f_{ \pm}^{0, R / A} / \zeta_{2}, \\
& \tilde{F}_{ \pm}^{\prime R / A}=\tilde{f}_{ \pm}^{0, R / A} / \zeta_{1}, \quad \tilde{P}_{ \pm}^{\prime R / A}=\tilde{f}_{ \pm}^{0, R / A} / \zeta_{2}
\end{aligned}
$$

with $\zeta_{1}=g_{+}^{0, R}+g_{-}^{0, A}$ and $\zeta_{2}$ is obtained from $\zeta_{1}$ by exchanging $-\leftrightarrow+$. The Keldysh-like matrices are

$$
\begin{aligned}
& G_{ \pm}^{K}=\zeta_{ \pm} \phi_{ \pm}^{0} / \zeta_{1}, \quad Q_{ \pm}^{K}=\zeta_{ \pm} \phi_{ \pm}^{0} / \zeta_{2}, \\
& F_{ \pm}^{\prime K}=\left[F_{ \pm}^{\prime R}-F_{ \pm}^{A}\right] \phi_{ \pm}^{0}, \quad P_{ \pm}^{\prime K}=\left[P_{ \pm}^{\prime R}-F_{ \pm}^{A}\right] \phi_{ \pm}^{0}, \\
& \tilde{F}_{ \pm}^{\prime K}=\left[\tilde{F}_{ \pm}^{R}-\tilde{F}_{ \pm}^{\prime A}\right] \phi_{ \pm}^{0}, \quad \tilde{P}_{ \pm}^{\prime K}=\left[\tilde{F}_{ \pm}^{R}-\tilde{P}_{ \pm}^{\prime A}\right] \phi_{ \pm}^{0},
\end{aligned}
$$

where $\phi_{ \pm}^{0}$ is the occupation associated with $g_{ \pm}^{0}$ and $\zeta_{ \pm}=$ $g_{ \pm}^{0, R}-g_{ \pm}^{0, A}$.

We have thus shown that a small tilt angle produces spin-flip scattering that combined with Andreev scattering processes leads to spinful anomalous Green's functions, $\delta f_{\uparrow / \downarrow}^{R, K, A}$, that in turn generate off-diagonal, spinful normal Green's functions, $\delta g_{\uparrow / \downarrow}^{R, K, A}$. Consequently, the spin current given by Eq. (14) is nonzero as a result of the induced equal spin triplet anomalous Green's functions.
${ }^{1}$ M. N. Baibich, J. M. Broto, A. Fert, F. Nguyen, Van Dau, F. Petroff, P. Etienne, G. Creuzet, A. Friederich, and J. Chazelas, Phys. Rev. Lett. 61, 2472 (1988).

${ }^{2}$ G. Binasch, P. Grünberg, F. Saurenbach, and W. Zinn, Phys. Rev. B 39, 4828 (1989).

${ }^{3}$ S. A. Wolf, D. D. Awschalom, R. A. Buhrman, J. M. Daughton, S. von Molnár, M. L. Roukes, A. Y. Chtchelkanova, and D. M. Trege, Science 294, 1488 (2001).

${ }^{4}$ M. Elbing, R. Ochs, M. Koentopp, M. Fischer, C. von Hänisch, F. Weigend, F. Evers, H. B. Weber, and M. Mayor, PNAS 102, 8815 (2005).

${ }^{5}$ J. Park, A. N. Pasupathy, J. I. Goldsmith, C. Chang, Y. Yaish, J. R. Petta, M. Rinkoski, J. P. Sethna, H. D. Abruña, P. L. McEuen, and D. C. Ralph, Nature (London) 417, 722 (2002).

${ }^{6}$ E. Osorio, T. Bjørnholm, J.-M. Lehn, M. Ruben, and H. S. J. van der Zant, J. Phys.: Condens. Matter 20, 374121 (2008).

${ }^{7}$ L. H. Yu, Z. K. Keane, J. W. Ciszek, L. Cheng, J. M. Tour, T. Baruah, M. R. Pederson, and D. Natelson, Phys. Rev. Lett. 95, 256803 (2005).

${ }^{8}$ L. Bogani and W. Wernsdorfer, Nat. Mat. 7, 179 (2008).

${ }^{9}$ G. Christou, D. Gatteschi, D. N. Hendrickson, and R. Sessoli, MRS Bulletin 25, 66 (2000).

${ }^{10}$ W. Wernsdorfer and R. Sessoli, Science 284, 133 (1999).

${ }^{11}$ H. B. Heersche, Z. de Groot, J. A. Folk, H. S. J. van der Zant, C. Romeike, M. R. Wegewijs, L. Zobbi, D. Barreca, E. Tondello, and A. Cornia, Phys. Rev. Lett. 96, 206801 (2006).

${ }^{12}$ M.-H. Jo, J. E. Grose, K. Baheti, M. M. Deshmukh, J. J. Sokol, E. M. Rumberger, D. N. Hendrickson, J. R. Long, H. Park, and D. C. Ralph, Nano Lett. 6, 2014 (2006).

${ }^{13}$ J. J. Henderson, C. M. Ramsey, E. del Barco, A. Mishra, and G. Christou, J. Appl. Phys. 101, 09E102 (2007).

${ }^{14}$ J. E. Grose, E. S. Tam, C. Timm, M. Scheloske, B. Ulgut, J. J. Parks, H. D. Abruña, W. Harneit, and D. C. Ralph, Nat. Mater. 7, 884 (2008).

${ }^{15}$ A. S. Zyazin, J. W. G. van den Berg, E. A. Osorio, H. S. J. van der Zant, N. P. Konstantinidis, M. Leijnse, M. R. Wegewijs, F. May, W. Hofstetter, C. Danieli, and A. Cornia, Nano Lett. 10, 3307 (2010).
${ }^{16}$ N. Roch, R. Vincent, F. Elste, W. Harneit, W. Wernsdorfer, C. Timm, and F. Balestro, Phys. Rev. B 83, 081407(R) (2011).

${ }^{17}$ F. Haque, M. Langhirt, E. del Barco, T. Taguchi, and G. Christou, J. Appl. Phys. 109, 07B112 (2011).

${ }^{18}$ M. Urdampilleta, S. Klyatskaya, J.-P. Cleuziou, M. Ruben, and W. Wernsdorfer, Nat. Mat. 6, 185 (2011).

${ }^{19}$ S. Kahle, Z. Deng, N. Malinowski, C. Tonnoir, A. Forment-Aliaga, N. Thontasen, G. Rinke, D. Le, V. Turkowski, T. S. Rahman, S. Rauschenbach, M. Ternes, and K. Kern, Nano Lett. 12, 518 (2012).

${ }^{20}$ M. Eschrig, Phys. Today 64, 43 (2011).

${ }^{21}$ L. N. Bulaevskii, V. V. Kuzii, and A. A. Sobyanin, Pis'ma Zh. Eksp. Teor. Fiz. 25, 314 (1977) [JETP Lett. 25, 290 (1977)].

${ }^{22}$ V. V. Ryazanov, V. A. Oboznov, A. Yu. Rusanov, A. V. Veretennikov, A. A. Golubov, and J. Aarts, Phys. Rev. Lett. 86, 2427 (2001).

${ }^{23}$ T. Kontos, M. Aprili, J. Lesueur, F. Genêt, B. Stephanidis, and R. Boursier, Phys. Rev. Lett. 89, 137007 (2002).

${ }^{24}$ A. I. Buzdin, Rev. Mod. Phys. 77, 935 (2005).

${ }^{25}$ F. S. Bergeret, A. F. Volkov, and K. B. Efetov, Phys. Rev. Lett. 86, 3140 (2001).

${ }^{26}$ F. S. Bergeret, A. F. Volkov, and K. B Efetov, Rev. Mod. Phys. 77, 1321 (2005).

${ }^{27}$ M. Houzet and A. I. Buzdin, Phys. Rev. B 76, 060504(R) (2007).

${ }^{28}$ V. Braude and Yu. V. Nazarov, Phys. Rev. Lett. 98, 077003 (2007).

${ }^{29}$ M. Eschrig and T. Löfwander, Nat. Phys. 4, 138 (2008).

${ }^{30}$ R. S. Keizer, S. T. B. Goennenwein, T. M. Klapwijk, G. Miao, G. Xiao, and A. Gupta, Nature (London) 439, 825 (2006).

${ }^{31}$ T. S. Khaire, M. A. Khasawneh, W. P. Pratt, Jr., and N. O. Birge, Phys. Rev. Lett. 104, 137002 (2010).

${ }^{32}$ X. Waintal and P. W. Brouwer, Phys. Rev. B 63, 220407(R) (2001).

${ }^{33}$ X. Waintal and P. W. Brouwer, Phys. Rev. B 65, 054407 (2002).

${ }^{34}$ E. Zhao and J. A. Sauls, Phys. Rev. B 78, 174511 (2008).

${ }^{35}$ Z. Shomali, M. Zareyan, and W. Belzig, New J. Phys. 13, 083033 (2011).

${ }^{36}$ V. Braude and Ya. M. Blanter, Phys. Rev. Lett. 100, 207001 (2008).

${ }^{37}$ J. Michelsen, V. S. Shumeiko, and G. Wendin, Phys. Rev. B 77, 184506 (2008). 
${ }^{38}$ I. Petković, M. Aprili, S. E. Barnes, F. Beuneu, and S. Maekawa, Phys. Rev. B 80, 220502 (2009).

${ }^{39}$ S. E. Barnes, M. Aprili, I. Petković, and S. Maekawa, Supercond. Sci. Technol. 24, 024020 (2011).

${ }^{40}$ S. Mai, E. Kandelaki, A. F. Volkov, and K. B. Efetov, Phys. Rev. B 84, 144519 (2011).

${ }^{41}$ J. Linder and T. Yokoyama, Phys. Rev. B 83, 012501 (2011).

${ }^{42}$ C. Holmqvist, S. Teber, and M. Fogelström, Phys. Rev. B 83, 104521 (2011).

${ }^{43}$ S. Teber, C. Holmqvist, and M. Fogelström, Phys. Rev. B 81, 174503 (2010).

${ }^{44}$ A. Yu. Kasumov, K. Tsukagoshi, M. Kawamura, T. Kobayashi, Y. Aoyagi, K. Senba, T. Kodama, H. Nishikawa, I. Ikemoto, K. Kikuchi, V. T. Volkov, Yu. A. Kasumov, R. Deblock, S. Guéron, and H. Bouchiat, Phys. Rev. B 72, 033414 (2005).

${ }^{45}$ M. Houzet, Phys. Rev. Lett. 101, 057009 (2008).

${ }^{46}$ C. Bell, S. Milikisyants, M. Huber, and J. Aarts, Phys. Rev. Lett. 100, 047002 (2008).

${ }^{47}$ Y. Tserkovnyak, A. Brataas, and G. E. W. Bauer, Phys. Rev. Lett. 88, 117601 (2002); Y. Tserkovnyak, A. Brataas, G. E. W. Bauer, and B. I. Halperin, Rev. Mod. Phys. 77, 1375 (2005).

${ }^{48}$ J. X. Zhu and A. V. Balatsky, Phys. Rev. B 67, 174505 (2003).

${ }^{49}$ J. X. Zhu, Z. Nussinov, A. Shnirman, and A. V. Balatsky, Phys. Rev. Lett. 92, 107001 (2004).

${ }^{50}$ G. Eilenberger, Z. Phys. 214, 195 (1968).

${ }^{51}$ A. I. Larkin and Yu. N. Ovchinnikov, Zh. Eksp. Teor. Fiz. 55, 2262 (1968) [Sov. Phys. JETP 28, 1200 (1969)].

${ }^{52}$ G. M. Eliashberg, Zh. Eksp. Teor. Fiz. 61, 1254 (1971) [Sov. Phys. JETP 34, 668 (1972)].
${ }^{53}$ C. Caroli, R. Combescot, P. Nozieres, and D. Saint-James, J. Phys. C 4, 916 (1971).

${ }^{54}$ L. J. Buchholtz and D. Rainer, Z. Phys. B 35, 151 (1979).

${ }^{55}$ A. Martín-Rodero, F. J. García-Vidal, and A. Levy Yeyati, Phys. Rev. Lett. 72, 554 (1994); A. Levy Yeyati, A. Martín-Rodero, and F. J. García-Vidal, Phys. Rev. B 51, 3743 (1995).

${ }^{56}$ J. C. Cuevas and M. Fogelström, Phys. Rev. B 64, 104502 (2001).

${ }^{57}$ J. Kopu, M. Eschrig, J. C. Cuevas, and M. Fogelström, Phys. Rev. B 69, 094501 (2004).

${ }^{58}$ F. S. Bergeret, P. Virtanen, T. T. Heikkilä, and J. C. Cuevas, Phys. Rev. Lett. 105, 117001 (2010).

${ }^{59}$ F. S. Bergeret, P. Virtanen, A. Ozaeta, T. T. Heikkilä, and J. C. Cuevas, Phys. Rev. B 84, 054504 (2011).

${ }^{60}$ J.-D. Pillet, C. H. L. Quay, P. Morfin, C. Bena, A. Levy Yeyati, and P. Joyez, Nat. Phys. 6, 965 (2010).

${ }^{61}$ C. W. J. Beenakker, Phys. Rev. Lett. 67, 3836 (1991).

${ }^{62}$ V. S. Shumeiko, E. N. Bratus, and G. Wendin, Low Temp. Phys. 23, 181 (1997).

${ }^{63}$ J. W. Serene and D. Rainer, Phys. Rep. 101, 21 (1983).

${ }^{64}$ J. C. Slonczewski, J. Magn. Magn. Mater. 159, L1 (1996).

${ }^{65}$ L. Berger, Phys. Rev. B 54, 9353 (1996).

${ }^{66}$ T. Gilbert, IEEE Trans. Magn. 40, 3443 (2004).

${ }^{67}$ M. F. Goffman, R. Cron, A. Levy Yeyati, P. Joyez, M. H. Devoret, D. Esteve, and C. Urbina, Phys. Rev. Lett. 85, 170 (2000).

${ }^{68}$ E. Scheer, P. Joyez, D. Esteve, C. Urbina, and M. H. Devoret, Phys. Rev. Lett. 78, 3535 (1997).

${ }^{69}$ E. Scheer, W. Belzig, Y. Naveh, M. H. Devoret, D. Esteve, and C. Urbina, Phys. Rev. Lett. 86, 284 (2001).

${ }^{70}$ M. L. Della Rocca, M. Chauvin, B. Huard, H. Pothier, D. Esteve, and C. Urbina, Phys. Rev. Lett. 99, 127005 (2007). 